\title{
Desafios no uso de Dados Abertos Conectados na Educação Brasileira
}

\author{
Williams Alcantara ${ }^{1}$, Judson Bandeira ${ }^{1}$, Armando Barbosa ${ }^{1}$, André Lima ${ }^{1}$, \\ Thiago Ávila ${ }^{1}$, Ig Bittencourt ${ }^{1}$, Seiji Isotani ${ }^{2}$ \\ ${ }^{1}$ Núcleo de Excelência em Tecnologias Sociais (NEES) \\ Instituto de Computação - Universidade Federal de Alagoas (UFAL) \\ CEP 57.072-900 - Maceió - AL - Brasil \\ ${ }^{2}$ Laboratório de Computação Aplicada à Educação (CAEd) \\ Instituto de Ciências Matemáticas e de Computação - Universidade de São Paulo (USP) \\ CEP 13.566-590 - São Paulo - SP - Brasil \\ \{wla,jmb,abs, avtl, tjta, ig.ibert\}@ic.ufal.br, sisotani@icmc.usp.br
}

\begin{abstract}
The amount of data published on the web has grown in recent years. In Brazil, several government agencies have started the process of opening data to improve transparency and intensify the collaboration between population and government. In education, there are lots of data being generated every day. However, these data sets have problems that itself makes hard the enrichment and connection data in this scenario. This paper presents the challenges in application of Linked Open Data in Brazilian educational context. The challenges are organized under two perspectives: publication and consumption. The goal of this work is establish discussions about solutions to challenges.
\end{abstract}

Resumo. A quantidade de dados publicados na web tem crescido nos últimos anos. No Brasil, diversos órgãos públicos têm iniciado o processo de abertura de dados objetivando efetivar a transparência e intensificar a colaboração entre governo e população. Na educação, existe uma diversidade de dados sendo gerados todos os dias. Contudo, essas bases apresentam problemas que dificultam o enriquecimento e a conexão dos dados neste cenário. Este artigo apresenta os principais desafios na aplicação de Dados Abertos Conectados na educação brasileira. Os desafios estão organizados sob as perspectivas de publicação e consumo. O objetivo deste trabalho é estabelecer discussões que proponham soluções para os desafios.

\section{Introdução}

Com o crescimento da web e o uso massivo de tecnologias da informação, a quantidade de dados gerados e disponibilizados tem crescido exponencialmente. Esses dados estão, predominantemente, em formato não estruturado - o que limita sua descrição e reutilização por outras aplicações e pessoas. Além disso, devido à baixa qualidade dos dados disponibilizados, o processo de reutilização tem sido caro.

Atualmente, é visível a utilização da tecnologia para dar suporte à educação. Dessa forma, surgem várias abordagens para melhoria do aprendizado do aluno, 
como Jogos sérios [Susi et al. 2007], Gamificação [Deterding et al. 2011], Ambientes Virtuais de Aprendizagem [Wilson 1996] e Sistemas Tutores [Ma et al. 2014]. Contudo, como desenvolvedores estão lidando com a eficiência destes softwares, algumas preocupações podem surgir, como por exemplo, a conexão entre repositórios de objetos de aprendizagem e interoperabilidade de dados entre plataformas educacionais. Para esta conexão ser realizada, os pesquisadores exploram cada vez mais os benefícios de Dados Conectados e Web Semântica [Berners-Lee et al. 2001].

A publicação de dados tem gerado benefícios nas diversas áreas em que ocorre, como a transparência em órgãos governamentais que é utilizada como uma importante ferramenta no combate a corrupção e a ampliação da participação da sociedade no processo de desenvolvimento de novas soluções. Além do setor governamental, a educação também torna-se uma beneficiária, pois a grande quantidade de dados disponíveis pode auxiliar na tomada de decisão de professores e gestores escolares.

De acordo com [Bandeira et al. 2015], a publicação de dados abertos deve obedecer aos seguintes critérios: i) disponibilidade - a completude e a manutenção da base de dados são imprescindíveis para manter a qualidade de uma fonte de dados; ii) reúso - sob uma licença que permita a sua reutilização e redistribuição, requerendo apenas que ocorra sob a mesma licença; iii) participação universal qualquer pessoa está habilitada a usar, reusar e redistribuir, não havendo nenhum tipo de discriminação a uma pessoa ou um grupo; e iv) legível por máquina - a base deve ser publicada em formatos que facilitem a legibilidade pela máquina.

O consumo de dados abertos pode ser feito por públicos de diferentes perfis. Desenvolvedores tendem a utilizar APIs e Webservices, pois facilitam o consumo automatizado sob os dados. Por outro lado, pessoas que não são desenvolvedores preferem que a base esteja em formatos mais comuns, como PDF, CSV e DOC, pois são formatos legíveis para eles. O consumo de dados abertos agrega valor a bases de conhecimento com informações complementares sobre um determinado contexto, possibilitando a melhoria ou novas soluções para problemas cotidianos. Apesar dos benefícios, uma base de dados com baixa qualidade pode acarretar em problemas, como a baixa interoperabilidade e a duplicidade dos dados, além da grande quantidade em um mesmo conjunto (data set), dificultando o consumo.

Uma das alternativas encontradas pela comunidade Web para o problema da baixa qualidade de dados e da problemática de distribuição é a utilização de Dados Abertos Conectados (DAC) - são um conjunto de boas práticas para a publicação e consumo de dados abertos. Os princípios de DAC podem ser aplicados a vários contextos como saúde, segurança e educação [Bandeira et al. 2015].

Ao aplicar Dados Abertos Conectados em contextos educacionais, é possível: melhorar a qualidade de conteúdos publicados - através do uso de vocabulários que formalizam e descrevem os termos utilizados; melhorar a veracidade das informações - através de links para a fonte de dados; e agilizar o processo de recuperação de conteúdo educacional - através da conexão entre repositórios com conteúdo educacional. No Brasil, existem problemas em relação aos dados educacionais pela sua falta de completude, qualidade da informação e formatos de dados inadequados para reúso. Estes problemas criam barreiras para o desenvolvimento de novas soluções. 


\section{Trabalhos relacionados}

Em [Dietze et al. 2013], são apresentados desafios para aplicação de dados conectados na educação. Apesar das contribuições, o mesmo leva em consideração apenas os desafios encontrados pela comunidade de dados conectados, não considerando o contexto educacional.

Em [Atkins et al. 2007], é discutido sobre os desafios de se investir em Recursos Educacionais Abertos, como Sustentabilidade, Preservação do Acesso, Granularidade de objetos e Diversidade de Formatos, Questões de propriedade intelectual, Avaliação da Qualidade e Melhoria do Conteúdo, Infraestrutura de Computação e Comunicação e Impacto no Desenvolvimento de Países. Porém, percebe-se que este trabalho aborda mais as questões sobre dados abertos do que dados conectados.

Em [d'Aquin et al. 2013], é feita uma avaliação dos conjuntos de dados relacionados com educação, encontrando um certo nível de heterogeneidade entre esses conjuntos e mostra como uma integração entre os conjuntos pode melhorar a coesão da web de dados educacionais fazendo um alinhamento dos vocabulários utilizados nesses conjuntos de dados. Novamente, são mostradas soluções para apresentar melhorias sob a perspectiva computacional, e não educacional.

\section{Embasamento teórico}

Esta seção provê uma visão geral sobre Dados Abertos Conectados, apresentando um embasamento teórico suficiente para o entendimento deste trabalho.

\subsection{Dados abertos}

Segundo a Open Knowledge Foundation (OKFN) ${ }^{1}$, dados abertos devem ser usados, modificados e compartilhados por qualquer pessoa e para qualquer finalidade, exigindo-se apenas que seja preservada a fonte e compartilhado sob a mesma licença.

No âmbito governamental, os dados abertos são fundamentais para possibilitar transparência, participação e colaboração entre governo e sociedade, como ilustra a Figura 1.

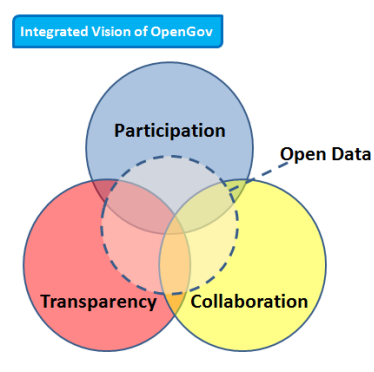

Figura 1. Open Gov

De acordo com [Handbook 2012], são características de dados abertos:

- Disponibilidade e Acesso: estar disponível como um todo, sob um custo razoável de reprodução e em um formato conveniente e modificável.

\footnotetext{
${ }^{1}$ http: //opendefinition.org
} 
- Reúso e Redistribuição: garantir a legibilidade por máquina, provida sob termos que permitam o reúso e redistribuição.

- Participação Universal: o uso dos dados pode ser exercido livremente por qualquer grupo ou pessoa.

A partir do uso de dados abertos surgem ferramentas como o $\mathrm{QEdu}{ }^{2}$, que reúsa dados abertos educacionais para prover a visualização de forma interativa, auxiliando na tomada de decisão aos gestores escolares e a sociedade para propor melhorias no sistema educacional, conforme ilustra a Figura 2.

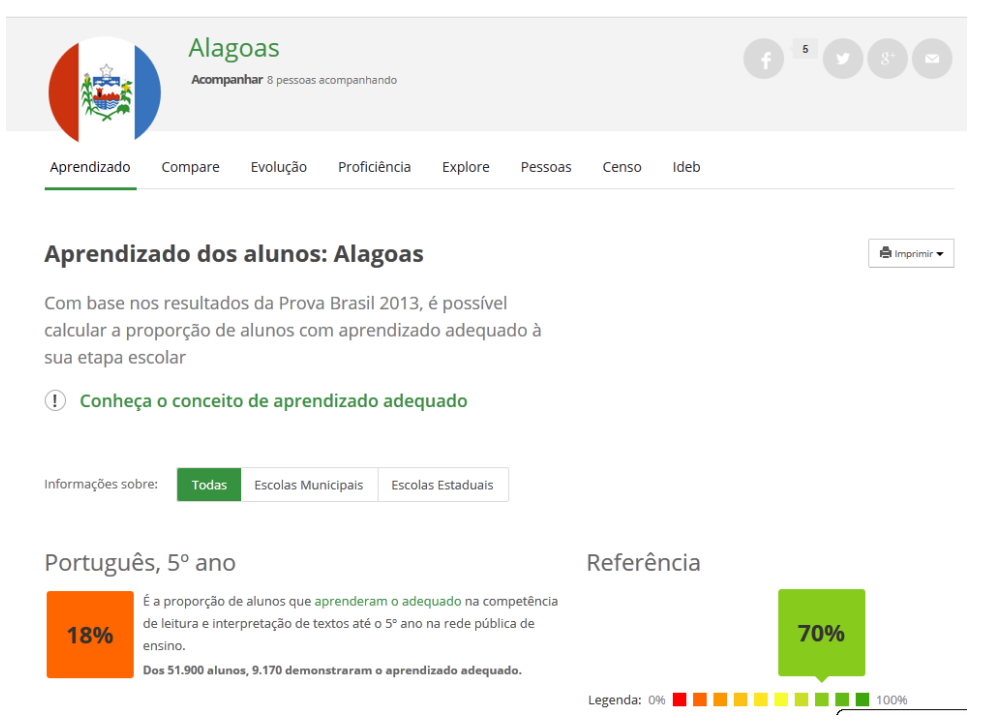

Figura 2. Resultado do estado de Alagoas na Prova Brasil 2013 [QEdu 2015]

\subsection{Dados abertos conectados}

A Web possui dados publicados em diversos formatos. Em sua maioria são formatos não estruturados e são adequados para facilitar o acesso e leitura para humanos e não são compreensíveis por máquina. Com o uso de Dados Conectados é possível que ambos consigam ler e interpretar os dados, visto que são um conjunto de boas práticas para publicar e consumir dados através da web [Bandeira et al. 2015]

Em [Berners-Lee 2011] são definidos os quatro princípios para publicação:

1. Use URIs para definir coisas;

2. Use HTTP URIs para que os dados possam ser encontrados por humanos e agentes na web;

3. Quando um dado for solicitado através de HTTP URIs, fornecer todas as informações sobre o mesmo, em um formato de dados estruturados utilizando padrões como RDF e SPARQL;

4. Incluir links para outras fontes de dados relacionados (usando URIs) para que seja possível obter mais informações.

De acordo com [Bandeira et al. 2015], os Dados Abertos Conectados são conjuntos de Dados Conectados que estão submetidos a uma licença aberta, que

\footnotetext{
${ }^{2}$ http://www .qedu.org.br
} 
permite o seu uso e distribuição livremente. Para medir a qualidade, [Berners-Lee 2011] propôs o sistema de classificação 5-estrelas, o qual é descrito abaixo.

^ Disponível na Web, em qualquer formato (por exemplo, uma imagem de um documento escaneado);

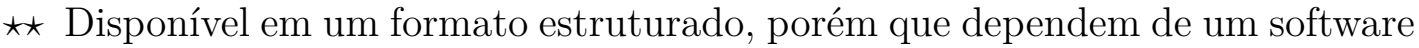
proprietário para a sua utilização (por exemplo, uma planilha do Excel ao invés de uma imagem escaneada de uma tabela);

$\star \star \star$ Disponível em um formato estruturado e não proprietário (por exemplo, CSV ao invés de Excel);

$\star \star \star \star$ Use padrões abertos recomendados pela W3C (RDF e SPARQL) para identificar recursos, de modo que as pessoas possam apontar para eles;

$\star \star \star \star \star$ Todos os itens acima se aplicam, e também adicionar links para outras bases de dados relacionados.

Ainda de acordo com [Berners-Lee 2011], o sistema de classificação 5-estrelas pode ser utilizado para medir a qualidade de uma base de Dados Conectados, desde que seja desconsiderado o requisito no qual o dado deve estar sob uma licença aberta.

\section{Desafios}

Conforme visto anteriormente, a abertura e enriquecimento de dados geram importantes benefícios. No entanto, os dados educacionais brasileiros apresentam problemas que dificultam a transformação para dados abertos conectados. Serão apresentados 5 desafios, os quais, estão sob duas perspectivas: publicação e consumo.

\subsection{Desafios na publicação de dados educacionais conectados}

A publicação de dados é uma atividade importante para o compartilhamento de informações, no contexto educacional isso pode gerar benefícios tanto no campo científico quanto no campo de negócio.

\subsubsection{Como estimular a publicação de dados educacionais em formato aberto?}

Com a crescente popularização do uso de dados abertos e conectados, a disponibilização de dados deve ser feita de forma que facilite a sua utilização.

A publicação de dados em formato proprietário, como o PDF, não é uma boa prática para o incentivo a sua reutilização. Além de não ser um formato estruturado ele restringe o acesso por pessoas ou empresas, sendo necessário adquirir pacotes de softwares proprietário para utilizá-lo. Além disso, os dados são publicados em formatos não estruturados, como TXT, DOC, ODT e PDF, dificultando a sua utilização [Wood et al. 2013]. Com o uso de dados estruturados há a organização dos dados em regras bem definidas, possibilitando a aplicação de filtros e consultas, agrupamento e extração de dados relevantes para os usuários [Almeida 2002].

O Brasil ainda reflete essa problemática, visto que o hábito de publicar dados em formato aberto não está disseminado. Os órgãos educacionais ainda não aderiram as práticas de publicação de dados abertos conectados, dificultando a transparência 
e a utilização dos dados no desenvolvimento de pesquisas, e consequentemente, na geração de melhorias em serviços e novas soluções.

Por exemplo, o Instituto Nacional de Matemática Pura e Aplicada (IMPA) e Instituto Nacional de Estudos e Pesquisas Educacionais Anísio Teixeira (INEP) disponibilizam bancos de questões da Olímpiada Brasileira de Matemática das Escolas Públicas (OBMEP) ${ }^{3}$ e do Exame Nacional do Ensino Médio (ENEM) ${ }^{4}$, respectivamente, no formato PDF e CSV.

A publicação em formato aberto e estruturado de bancos de questões de olímpiadas, facilitaria o desenvolvimento de soluções tecnológicas que reúnem questões de olímpiadas e exames nacionais em um único sistema que visa contribuir para o treinamento e capacitação dos alunos que se dedicam a esses exames.

Existem poucos casos de uso de dados abertos no Brasil, o que pode influenciar na relutância, por parte de órgãos responsáveis pela publicação, por ainda não terem adotado essas práticas e não ter acreditado no potencial de transparência e inovação que será gerado. Dessa forma, é necessário estimular e disseminar a cultura de publicação de dados educacionais em formato aberto.

O governo é um importante beneficiário da publicação de dados abertos e deve desenvolver ações que sejam efetivas para a sua implantação, assim como foi feito pelo governo dos Estados Unidos [Obama 2009]. Outra iniciativa importante é a qualificação de profissionais, apoiando a criação e manutenção do ecossistema de dados abertos, como o curso online ofertado por [Bittencourt and Isotani 2015] .

\subsubsection{Como publicar dados em diferentes formatos, independentemente da infraestrutura tecnológica utilizada?}

Em sua maioria, os órgãos publicam dados com pouca diversidade de formatos, limitando a variedade de agentes que podem reutilizá-los, seja de forma manual ou automatizada. O ideal é que os dados possam ser legíveis por diferentes públicos consumidores, que são: i) humanos: pessoas que somente buscam lê-los para conseguir informação para si, não desejando gerar novas soluções tecnológicas a partir deles. Assim, preferem que eles sejam publicados em formatos como TXT, PDF, DOC, ODT, XLS, pois facilitam a leitura e o processamento de forma manual; e ii) máquinas: pessoas que necessitam do processamento automatizado sob os dados, preferem que estejam em formatos como JSON, XML e RDF.

Os dados educacionais publicados, em sua maioria, atendem somente a um público específico formado por pessoas que buscam informações de forma AD HOC, ou seja, aquelas que não têm a intenção de produzir novos serviços ou aplicações.

Sendo assim, as necessidades desse público são atendidas por dados estatísticos publicados em formato PDF, permitindo uma boa visualização e identificação de seu conteúdo de forma manual. Contudo, é um formato proprietário não es-

\footnotetext{
${ }^{3}$ Disponível em http://www.obmep.org.br/banco.htm

${ }^{4}$ Disponível em http://portal.inep.gov.br/web/enem/edicoes-anteriores/provas-egabaritos
} 
truturado que impõe barreiras na utilização por agentes que consumem de forma automatizada.

A utilização e manutenção de sistemas legados em órgãos educacionais não possibilitam uma infraestrutura adequada para implantar o uso de APIs, permitindo a publicação de forma ampla e que facilite o consumo.

No Brasil, órgãos governamentais não possuem uma interface de comunicação ágil entre si, publicam dados relacionados como se fossem recursos distintos, não fazendo a referência necessária durante a publicação. A causa disso é a utilização de sistemas legados, que não conseguem se adaptar as novas tecnologias e novas demandas geradas pela sociedade. Com isso, os sistemas legados se tornam um empecilho à interoperabilidade e federalização de informações, e assim, as demandas geradas são impossibilitadas de serem ofertadas pelo governo em tempo hábil.

Um caminho para a solução desse desafio surge com a utilização de ferramentas que flexibilizem a interface entre o provimento e a disponibilização dos dados, podendo disponibilizá-los em diversos formatos que atendam aos diferentes públicos. O uso de vocabulários RDF para descrevê-los já atende a essa necessidade, pois eles podem ser utilizados em serializações como RDF/XML, RDF/JSON e turtle. Além disso, o uso de RDF implica na utilização de banco de dados semânticos como OWLIM e Virtuoso que permitem também a exportação dos dados em planilhas.

\subsubsection{Como estimular a publicação de dados educacionais conectados?}

A publicação de dados em formato aberto e estruturado não é o bastante para permitir que aplicações enriqueçam suas bases de conhecimento com eficiência. O ideal é que sejam publicados de acordo com o conjunto de boas práticas ${ }^{5}$ que permitem a interoperabilidade de dados na web através do uso de vocabulários RDF, facilitando a sua utilização por serviços automatizados para o consumo de dados.

No Brasil ainda são raras as iniciativas para a publicação de Dados Conectados. Nos principais órgãos publicadores de dados educacionais, ainda existem muitas bases em que não ocorre a publicação utilizando identificadores únicos, ou seja, bases em que os dados sejam referenciados em planilhas eletrônicas utilizando identificadores já definidos por setores especializados. Com essa carência, o custo de tempo e esforço são enormes, o que torna essa prática inviável.

Tomando como exemplo as bases de dados da OBMEP e do ENEM, que são importantes fontes de pesquisas para investigar e melhorar o desempenho de alunos e suas respectivas escolas. Nesses instrumentos de avaliação, supondo que ambas as bases de dados já estivessem conectadas, seria possível gerar aplicações para visualização de dados que relacionassem as escolas e os resultados obtidos nessas avaliações. Além disso, seria possível avaliar o desempenho em séries históricas com maior facilidade, auxiliando na tomada de decisão dos gestores escolares. Porém, isso não é possível de ser realizado atualmente, pois a base da OBMEP foi publicada no formato PDF e a do ENEM no formato CSV, e não utilizam as boas práticas de Dados Abertos Conectados, o que dificulta a interoperabilidade desses dados.

\footnotetext{
${ }^{5}$ Disponível em http://www.w3.org/TR/ld-bp/
} 
Dados Conectados permitiriam o compartilhamento de um vocabulário comum onde ambas as bases poderiam representar um mesmo recurso de forma idêntica, e também, permitiria a interoperabilidade de dados com maior consistência.

As inconsistências são problemas encontrados com frequência ao tentar conectar diferentes bases de dados educacionais brasileiras, é possível encontrar endereços de uma mesma escola escritos de duas maneiras diferentes. Isso pode ser solucionado com a atuação da gestão escolar, que é a responsável por fornecer tais dados. Os endereços devem ser referenciados conforme foram descritos pelo IBGE, e assim, evitando interpretações duvidosas sob os dados. Além disso, é importante a criação e manutenção de um banco de dados educacional aberto e conectado sendo disponibilizado através de Endpoint SPARQL, que permite usufruir de todos os benefícios da federalização em grandes bases de conhecimento [Sage and Cuppan 2001].

\subsection{Desafios no consumo de dados educacionais conectados}

O uso dos dados tem contribuido para a inovação em soluções educacionais, enriquecendo bases de conhecimento, e gerando soluções com qualidade.

\subsubsection{Como aplicar dados conectados a repositórios educacionais aber- tos?}

O uso de Repositórios Educacionais Abertos (REA) pode auxiliar no processo de ensino-aprendizagem. Escolas podem preparar materiais didáticos e compartilhá-los abertamente, possibilitando que os alunos tenham maior flexibilização no acesso e ainda dispor de outras fontes para consultar sobre assuntos relacionados.

Os REAs auxiliam o professor na obtenção do material didático utilizado para preparar o seu plano de aulas, e possibilita que o aluno usufrua de uma vasta fonte de materiais, utilizando-os como complemento de estudo ao ensino presencial. No entanto, dentre tantas fontes de materiais disponíveis e com a possibilidade de que qualquer pessoa está permitida a publicar conteúdo nesses repositórios, é um problema para que alunos e professores tenham ciência sobre quais fontes dos recursos publicados são mais confiáveis. Além disso, um REA pode não dispor de um bom material didático, surgindo a necessidade de que alunos e professores migrem para outros repositórios de recursos educacionais em busca de materiais relacionados. Ao fazer isso, surge outro problema, pois além de encontrarem outras fontes de materiais, podem encontrar materiais repetidos de outros repositórios tornando-se um processo muito complicado para que eles filtrem materiais didático com qualidade.

A duplicidade dos dados prejudica a efetividade do REA, pois a busca por recursos torna-se ineficiente, desestimulando os alunos e professores a utilizarem. Com a aplicação de dados conectados é possível contornar esse problema, visto que será possível identificar os recursos iguais que foram publicados em fontes diferentes, e assim, produzir um sistema de recomendação com maior confiabilidade e qualidade. 


\subsubsection{Como interoperar sistemas educacionais?}

Sistemas educacionais online, como os MOOCs, disponibilizam cursos online, disseminando conhecimento em larga escala, capacitando pessoas e diversificando o processo de ensino-aprendizagem [Yuan et al. 2013]. Nessas plataformas existem sistemas de recomendação que traçam o perfil do aluno e adaptam o curso para ele, explorando questões relacionadas aos assuntos em que ele possui maior dificuldade.

No Brasil, sistemas como VEDUCA ${ }^{6}$ e MeuTutor ${ }^{7}$ têm dados em comum, como dados de alunos, questões, textos, vídeos, entre outros. Apesar de possuírem uma grande base educacional, eles não compartilham padrões para representála, causando heterogeneidade e dificultando a interoperabilidade entre eles. Dessa forma, ao conectá-los pode ocorrer duplicação de dados, já que não utilizam padrões existentes para representar os dados. A utilização de Dados Abertos Conectados implica do uso de vocabulários RDF e permite que os sistemas compartilhem um modelo de representação. Consequentemente, a utilização de identificadores únicos (URIs) para cada recurso diminui a possibilidade de haver duplicação na base.

Para facilitar a interoperabilidade de dados entre tais sistemas é importante que eles sejam desenvolvidos seguindo a especificação de um sistema baseado em ontologias, o que possibilita enriquecimento semântico a base de conhecimento.

\section{Conclusão}

Este artigo apresentou os desafios na aplicação de Dados Abertos Conectados na educação. Percebe-se que a cultura de publicá-los, no Brasil, praticamente não existe. Os benefícios são desde a transparência até a colaboração entre população e governo. O Brasil ainda não dispõe de uma infraestrutura adequada para permitir tais acontecimentos, principalmente quando se trabalha com dados educacionais, visto que os setores que os detém ainda os publicam em formato proprietário e não estruturado, inviabilizando a sua reutilização. Assim, investigações acerca desses desafios podem contribuir para a disseminação da cultura de publicação e consumo de Dados Abertos Conectados na Educação Brasileira. A educação é um importante meio para o desenvolvimento de uma nação, tanto social quanto econômico, por isso espera-se que o processo de desenvolvimento se inicie por ela, sendo necessário maior envolvimento nas mudanças tecnológicas.

\section{Agradecimentos}

Agradecemos ao CNPq, NIC.br e Ceweb.br pelo apoio e fomento às pesquisas.

\section{Referências}

Almeida, M. B. (2002). Uma introdução ao xml, sua utilização na internet e alguns conceitos complementares. Ciência da Informação, 31(2):5-13.

Atkins, D. E., Brown, J. S., and Hammond, A. L. (2007). A review of the open educational resources (OER) movement: Achievements, challenges, and new opportunities. Creative common.

\footnotetext{
${ }^{6}$ http://www.veduca.com.br/

${ }^{7}$ http://www.meututor.com.br/
} 
Bandeira, J. M., Alcantara, W. L., Barbosa Sobrinho, A., Ávila, T. J. T., Bittencourt, I. I., and Isotani, S. (2015). Dados Abertos Conectados. III Simpósio Brasileiro de Tecnologia da Informação.

Berners-Lee, T. (2011). Linked data-design issues (2006). Disponível em: http: //www. w3. org/DesignIssues/LinkedData. html.

Berners-Lee, T., Hendler, J., Lassila, O., et al. (2001). The semantic web. Scientific american, 284(5):28-37.

Bittencourt, I. I. and Isotani, S. (2015). Dados abertos conectados. Escola de Políticas Públicas. Disponível em: http://www.ep.org.br/curso/58.

d'Aquin, M., Adamou, A., and Dietze, S. (2013). Assessing the educational linked data landscape. In Proceedings of the 5th Annual ACM Web Science Conference, pages 43-46. ACM.

Deterding, S., Dixon, D., Khaled, R., and Nacke, L. (2011). From game design elements to gamefulness: defining gamification. In Proceedings of the 15th International Academic MindTrek Conference: Envisioning Future Media Environments, pages 9-15. ACM.

Dietze, S., Sanchez-Alonso, S., Ebner, H., Qing Yu, H., Giordano, D., Marenzi, I., and Pereira Nunes, B. (2013). Interlinking educational resources and the web of data: A survey of challenges and approaches. Program, 47(1):60-91.

Handbook, O. D. (2012). What is open data? URL: http: // opendatahandbook. org/guide/en/what-is-open-data/.

Ma, W., Adesope, O. O., Nesbit, J. C., and Liu, Q. (2014). Intelligent tutoring systems and learning outcomes: A meta-analysis. American Psychological Association.

Obama, B. (2009). Memorandum on transparency and open government. Disponivel em: http://www. whitehouse. gov/sites/default/files/omb/ assets/memoranda \_2010/m10-06.pdf.

QEdu (2015). Aprendizado dos alunos: Alagoas. Disponível em http://www.qedu . org.br/estado/102-alagoas/aprendizado. Acessado em 20/05/2015.

Sage, A. P. and Cuppan, C. D. (2001). On the systems engineering and management of systems of systems and federations of systems. Information, Knowledge, Systems Management, 2(4):325-345.

Susi, T., Johannesson, M., and Backlund, P. (2007). Serious games: An overview. Institutionen för kommunikation och information.

Wilson, B. G. (1996). Constructivist learning environments: Case studies in instructional design. Educational Technology.

Wood, D., Zaidman, M., Ruth, L., and Hausenblas, M. (2013). Linked Data Structured Data on the Web. Manning Publications Co.

Yuan, L., Powell, S., and CETIS, J. (2013). Moocs and open education: Implications for higher education. Cetis White Paper. 